\title{
CNS-PNETs with C19MC amplification and/or LIN28 expression comprise a distinct histogenetic diagnostic and therapeutic entity
}

\author{
Tara Spence • Patrick Sin-Chan • Daniel Picard • Mark Barszczyk • Katharina Hoss • Mei Lu • Seung-Ki Kim • \\ Young-Shin Ra $\cdot$ Hideo Nakamura $\cdot$ Jason Fangusaro $\cdot$ Eugene Hwang $\cdot$ Erin Kiehna $\cdot$ Helen Toledano $\cdot$ Yin Wang $\cdot$ \\ Qing Shi • Donna Johnston · Jean Michaud • Milena La Spina • Anna Maria Buccoliero • Dariusz Adamek • \\ Sandra Camelo-Piragua $\cdot$ V. Peter Collins $\cdot$ Chris Jones $\cdot$ Nabil Kabbara $\cdot$ Nawaf Jurdi $\cdot$ Pascale Varlet $\cdot$ \\ Arie Perry • David Scharnhorst · Xing Fan · Karin M. Muraszko • Charles G. Eberhart • Ho-Keung Ng • \\ Sridharan Gururangan · Timothy Van Meter · Marc Remke · Lucie Lafay-Cousin · Jennifer A. Chan · \\ Nongnuch Sirachainan • Scott L. Pomeroy • Steven C. Clifford • Amar Gajjar • Mary Shago • William Halliday • \\ Michael D. Taylor · Richard Grundy $\cdot$ Ching C. Lau $\cdot$ Joanna Phillips $\cdot$ Eric Bouffet $\cdot$ Peter B. Dirks $\cdot$ \\ Cynthia E. Hawkins $\cdot$ Annie Huang
}

Received: 26 November 2013 / Revised: 1 May 2014 / Accepted: 2 May 2014 / Published online: 20 May 2014

(C) The Author(s) 2014. This article is published with open access at Springerlink.com

\begin{abstract}
Amplification of the C19MC oncogenic miRNA cluster and high LIN28 expression has been linked to a distinctly aggressive group of cerebral CNS-PNETs (group 1 CNS-PNETs) arising in young children. In this study, we sought to evaluate the diagnostic specificity of C19MC and LIN28, and the clinical and biological spectra of $C 19 M C$
\end{abstract}

T. Spence, P. Sin-Chan and D. Picard are co-first authors.

Electronic supplementary material The online version of this article (doi:10.1007/s00401-014-1291-1) contains supplementary material, which is available to authorized users.

T. Spence $\cdot$ P. Sin-Chan $\cdot$ D. Picard $\cdot$ K. Hoss $\cdot$ M. Lu $\cdot$

E. Bouffet $\cdot$ A. Huang $(\triangle)$

Division of Hematology-Oncology, Department of Pediatrics,

The Hospital for Sick Children, Arthur and Sonia Labatt Brain

Tumor Research Centre, Peter Gilgan CRL,686 Bay Street, 17th

Floor, 179712, Toronto, ON M5G0A4, Canada

e-mail: annie.huang@sickkids.ca

T. Spence $\cdot$ P. Sin-Chan · C. E. Hawkins $\cdot$ A. Huang

Department of Laboratory Medicine and Pathobiology,

University of Toronto, Toronto, ON, Canada

M. Barszczyk · W. Halliday · C. E. Hawkins

Department of Pathology, The Hospital for Sick Children,

Toronto, ON, Canada

\section{S.-K. Kim}

Department of Neurosurgery, Seoul National University

Children's Hospital, Seoul, South Korea

Y.-S. Ra

Department of Neurosurgery, Asan Medical Center, Seoul,

South Korea amplified and/or LIN28+ CNS-PNETs. We interrogated 450 pediatric brain tumors using FISH and IHC analyses and demonstrate that $C 19 M C$ alteration is restricted to a sub-group of CNS-PNETs with high LIN28 expression; however, LIN28 immunopositivity was not exclusive to CNS-PNETs but was also detected in a proportion of other malignant pediatric brain tumors including rhabdoid brain tumors and malignant gliomas. C19MC amplified/LIN28+ group 1 CNS-PNETs arose predominantly in children $<4$ years old; a majority arose in the cerebrum but $24 \%$ (13/54) of tumors had extra-cerebral origins. Notably,

\section{H. Nakamura}

Department of Neurosurgery, Kumamoto University,

Kumamoto, Japan

J. Fangusaro

Division of Pediatric Hematology/Oncology and Stem Cell

Transplantation, Children's Memorial Hospital,

Chicago, IL, USA

E. Hwang

Center for Cancer and Blood Disorders, Children's National

Medical Center, Washington, DC, USA

E. Kiehna

Department of Neurosurgery, Children's Hospital of Los Angeles,

Los Angeles, CA, USA

H. Toledano

Oncology Department, Schneider Hospital, Petach Tikva, Israel

Y. Wang

Department of Neuropathology Huashan Hospital, Fudan

University, Shanghai, China 
group 1 CNS-PNETs encompassed several histologic classes including embryonal tumor with abundant neuropil and true rosettes (ETANTR), medulloepithelioma, ependymoblastoma and CNS-PNETs with variable differentiation. Strikingly, gene expression and methylation profiling analyses revealed a common molecular signature enriched for primitive neural features, high LIN28/LIN28B and DNMT3B expression for all group 1 CNS-PNETs regardless of location or tumor histology. Our collective findings suggest that current known histologic categories of CNS-PNETs which include ETANTRs, medulloepitheliomas, ependymoblastomas in various CNS locations, comprise a common molecular and diagnostic entity and identify inhibitors of the LIN28/let7/PI3K/mTOR axis and DNMT3B as promising therapeutics for this distinct histogenetic entity.

\section{Introduction}

Primitive neuroectodermal tumors of the central nervous system (CNS-PNET) are a heterogeneous group of pediatric neoplasms composed of poorly differentiated neuroepithelial cells with varying degrees of divergent neural, astrocytic and ependymal differentiation. According to the current WHO CNS tumor working classification,

\section{Q. Shi}

Department of Pathology, Shanghai Children's Hospital Affiliated Shanghai Jiao Tong University School of Medicine, Shanghai, China

D. Johnston

Department of Pediatrics, Children's Hospital of Eastern Ontario, Ottawa, ON, Canada

\section{J. Michaud}

Department of Pathology and Laboratory Medicine, Children's Hospital of Eastern Ontario, Ottawa, ON, Canada

M. La Spina

Paediatric Haematology and Oncology Division, University of Catania, Sicily, Italy

\section{A. M. Buccoliero}

Pathology Unit Meyer Children's Hospital, Florence, Italy

\section{Adamek}

Department of Pathomorphology, Jagiellonian University

Medical College, Krakow, Poland

S. Camelo-Piragua

Department of Pathology, University of Michigan, Ann Arbor,

MI, USA

\section{Peter Collins}

Department of Pathology, University of Cambridge,

Cambridge, UK
CNS-PNETs are grouped into several histologic categories: CNS neuroblastoma/ganglioneuroblastoma, medulloepithelioma (MEP), ependymoblastoma (EPB) and classical CNS-PNET (PNET-NOS) [14]. In 2000, Eberhart et al. [4] described a new CNS-PNET variant arising primarily in infancy, which displayed histological features of both neuroblastoma and EPB, and were distinguished by the presence of true and pseudo-rosettes on a background of abundant neuropil. These tumors, termed 'embryonal tumors with abundant neuropil and true rosettes' (ETANTRs), correlated with very poor patient prognosis with a mortality rate of $76 \%$ and a median survival of 9 months $[1,5,21]$.

Li et al. [13] first reported C19MC amplification was enriched in cerebral CNS-PNETs with variant histologic features including tumors called ETANTR, MEP, EPB and PNETs with atypical features, thus suggesting that these conventional histologic sub-classes may represent closely related molecular entities. Indeed, Korshunov et al. [10] reported C19MC amplification in 37/40 tumors with a histologic diagnosis of ETANTR or EPB. In addition, C19MC amplification has been reported in tumors with mixed features of ETANTR and MEP [2, 16]. Subsequent studies demonstrated that upregulation of the RNA-binding pluripotency gene, LIN28 $[11,17]$, correlated closely with $C 19 M C$ amplification thus suggesting that LIN28 may represent an attractive immunodiagnostic marker for this distinct molecular sub-group of

\section{Jones}

Department of Paediatric Molecular Pathology, Institute of Cancer Research, Sutton, UK

N. Kabbara

Division of Pediatric Hematology Oncology, Rafic Hariri

University Hospital, Beirut, Lebanon

N. Jurdi

Department of Pathology and Laboratory Medicine, American

University of Beirut Medical Center, Beirut, Lebanon

P. Varlet

Medical and Department of Neuropathology, Sainte-Anne

Hospital, University Paris V Descartes, Paris, France

A. Perry $\cdot$ J. Phillips

Department of Pathology and Laboratory Medicine, University

of California, San Francisco, CA, USA

D. Scharnhorst

Department of Pathology, Children's Hospital Central California, Madera, CA, USA

X. Fan $\cdot$ K. M. Muraszko

Department of Neurosurgery, University of Michigan Medical

School, Ann Arbor, MI, USA

C. G. Eberhart

Division of Pathology, John Hopkins University School of Medicine, Baltimore, MD, USA 
cerebral CNS-PNETs. However, the relative diagnostic significance of C19MC and LIN28, and the molecular and therapeutic relationship of these different histologic sub-classes of CNS-PNETs remain to be completely elucidated.

To identify relevant therapeutic pathways for these tumors, we sought in this study to first evaluate the diagnostic specificity of C19MC amplification and LIN28 expression for CNS-PNETs, and define the histopathologic and clinical features of CNS-PNETs with C19MC amplification and/or LIN28 expression. We compared global gene expression and methylation data from C19MC amplified and/or LIN28+ CNS-PNETs with various histologic diagnostic labels and anatomic locations, and investigated pharmacologic inhibitors of LIN28/let-7/mTOR signaling and DNMT3B on growth of a novel cell line derived from a non-C19MC amplified group 1 CNS-PNET.

\section{Materials and methods}

Tumor and nucleic acid samples

Tumor specimens and clinical information were collected with consent as per protocols approved by Hospital Research Ethics Board at participating institutions. A total of 450 primary pediatric brain tumors with various histologic diagnoses-103 CNS-PNETs, 45 atypical rhabdoid teratoid tumors (ATRTs), 128 medulloblastomas (MBs), 105 ependymomas (EPNs), 50 high-grade gliomas (HGGs)

\section{H.-K. Ng}

Department of Anatomical and Cellular Physiology, Chinese

University of Hong Kong, Hong Kong, China

S. Gururangan

Department of Pediatrics, Duke University School of Medicine, Durham, NC, USA

\section{T. Van Meter}

Department of Pediatrics, Virginia Commonwealth University, Richmond, VA, USA

M. Remke · M. D. Taylor · P. B. Dirks

Division of Neurosurgery, Arthur and Sonia Labatt Brain Tumor Research Centre, The Hospital for Sick Children, Toronto, ON, Canada

L. Lafay-Cousin

Department of Pediatric Oncology, Alberta Children's Hospital, Calgary, AB, Canada

\section{J. A. Chan}

Department of Pathology and Laboratory Medicine, University of Calgary, Calgary, AB, Canada

N. Sirachainan

Departments of Pediatrics, Faculty of Medicine, Ramathibodi

Hospital, Bangkok, Thailand and 20 choroid plexus carcinomas (CPCs) were examined in this study (Supplemental Table 1). All ATRTs diagnoses were confirmed for genetic alterations of SMARCBI/INII by Multiplex Ligation mediated PCR and/or targeted gene sequencing and for loss of SMARCB1/INI1/BAF47 protein expression by immunostaining. Of the 54 group 1 CNSPNETs examined for detailed histopathologic correlates (Table 1 and Supplemental Table 2), 33 were previously reported [13, 17]; and 21 additional tumors with a diagnosis of CNS-PNET, ETANTR or embryonal tumor with multilayered rosettes (ETMR), MEP and EPB were collected for this study. Three tumors with an initial diagnosis of EPB were histologically reviewed and re-classified as ETANTR (PNET 5, 39 and 54). Tumor DNA and RNA were extracted by standard methods, quantified using NanoDrop Analyzer, and analyzed using the Illumina HT-12 v4 gene expression and $450 \mathrm{~K}$ Methylation arrays and OmniQuad 2.5 M SNP genotyping arrays (http://www.illumina .com) to generate gene expression, methylation, and copy number profiles. Methylation profiling was performed at The Centre for Innovation at Genome Quebec; all other analyses were performed at The Centre of Applied Genomics (TCAG) at the Hospital for Sick Children.

Histology, immunohistochemistry and fluorescence in situ hybridization

Hematoxylin and eosin ( $\mathrm{H}$ and $\mathrm{E})$ staining was performed using standard protocols. Fluorescence in situ hybridization

\section{S. L. Pomeroy}

Department of Neurology, Children's Hospital Boston, Boston, MA, USA

S. C. Clifford

Northern Institute for Cancer Research, Newcastle University, Newcastle Upon Tyne, UK

A. Gajjar

Neuro-oncology Division, St Jude Children's Research Hospital, Memphis, TN, USA

M. Shago

Department of Pediatric Laboratory Medicine, The Hospital for Sick Children, Toronto, ON, Canada

R. Grundy

Children's Brain Tumor Research Centre, Queen's Medical

Centre University of Nottingham, Nottingham, UK

C. C. Lau

Texas Children's Cancer Center, Baylor College of Medicine, Houston, TX, USA 
Table 1 Histopathologic and clinical features of C19MC amplified/LIN28+ CNS-PNETs

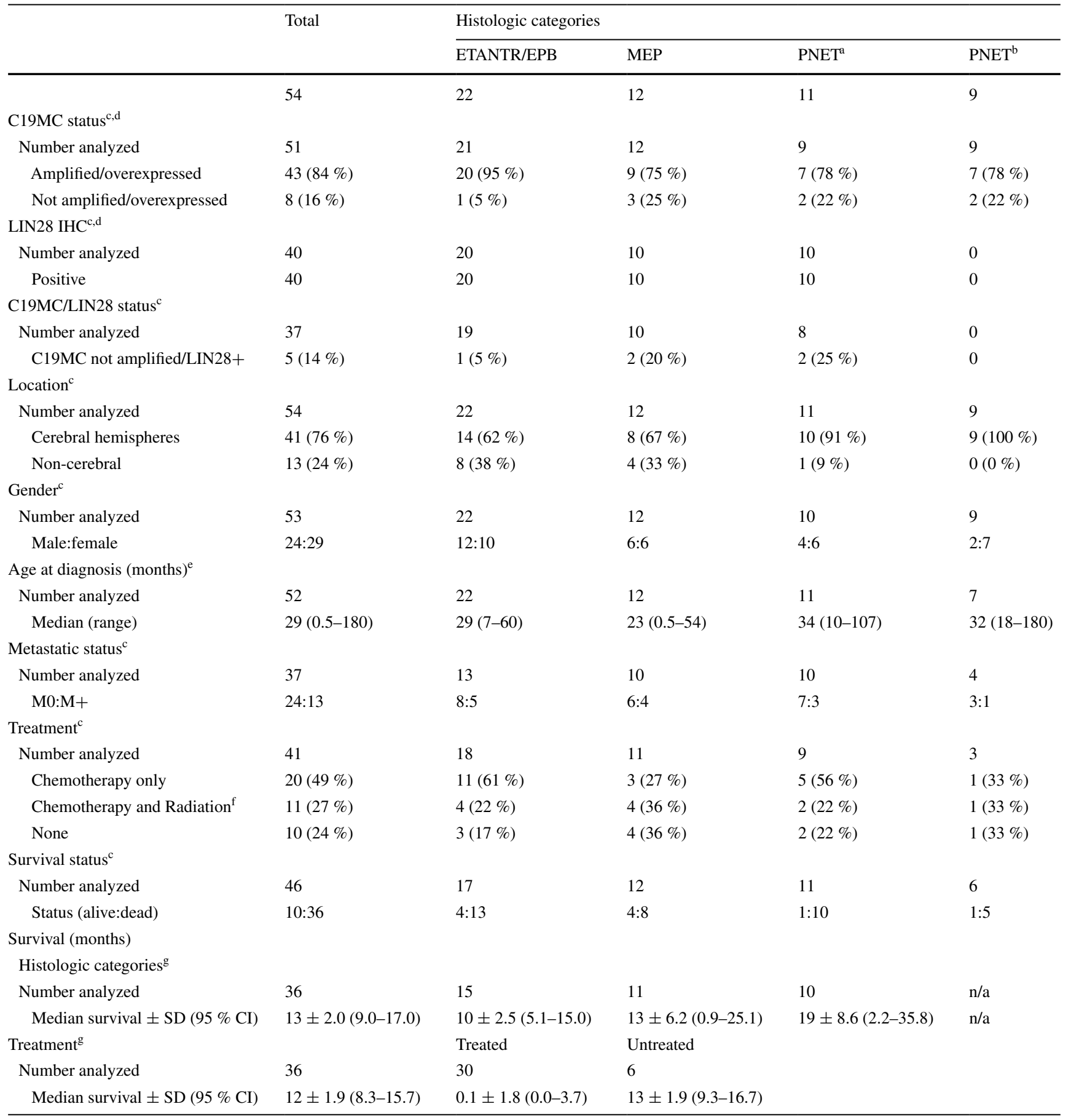

ETANTR embryonal tumor with abundant neuropil and true rosettes, EPB ependymoblastoma, $M E P$ medulloepithelioma, $P N E T$ primitive neuroectodermal tumor, $M 0$ no metastasis, $M+\mathrm{M} 1-\mathrm{M} 3$ metastasis as per Chang criteria

a Includes PNET with ependymal and anaplastic features

b Only institutional pathology diagnosis available

c Pearson Chi-Square

d Subset of tumors analyzed on TMAs (including 5/50 for C19MC FISH and 7/40 for LIN28 IHC)

e Kruskal-Wallis Test

f Includes one patient treated with radiation only

g Log-Rank (Mantel-Cox) test 
(FISH) and immunohistochemistry (IHC) were performed on 5- $\mu \mathrm{m}$ formalin-fixed paraffin-embedded (FFPE) sections for individual tumors or tissue microarrays (TMA). TMA construction was as previously detailed [18]. Tumor tissue blocks and corresponding slides were reviewed by an experienced neuropathologist $(\mathrm{CH})$ for diagnostic accuracy and adequacy of tissues; samples that had extensive necrosis or $<60 \%$ tumor content were discarded. Representative tumor areas were identified, and three 1-mm tissue cores were selected with the goal of obtaining a sampling accuracy $>95 \%$ for each tumor represented on the TMA [6, 8]. A variety of tissues including liver, fetal cerebellum, placenta, breast carcinoma, and basal cell carcinoma were included on each array to serve as internal controls for various immuno-stains.

For FISH analyses, pre-labeled BAC probes (http://www. tcag.ca/cytogenomics) mapping to chr19q13.42 (RP11381E3: 162, $225 \mathrm{bp}$ ) and an internal control chr19p13.11 locus (RP11-451E20: 165,783 bp) were used as described previously [13]. For IHC analyses, a heat-induced antigen retrieval process was used, followed by blocking endogenous peroxidase and biotin. Primary anti-LIN28 (\#3978) and anti-phospho-S6 (Ser240/244) (\#5364) antibodies were purchased from Cell Signaling Technology (Boston, MA, USA) and anti-DNMT3B (ab13604) was purchased from Abcam (Toronto, ON, CA). Antibody reactivity was visualized using the VectaStain ABC detection kit, Vector Laboratories (Burlingame, CA, USA).

For each protein, cytoplasmic and/or nuclear immunopositivity was visually scored based on both strength $(0,1$, $2,3-3+)$ and distribution $(<25,25-50,51-75$, and $>75 \%$ of tumor cells). Only tumors with strong staining (3-3+) in $>25 \%$ of tumor cells were considered to be positive. Tumors with FFPE slides were scored based on analyses of the whole tumor section, while tumors on TMAs were assessed on the basis of staining patterns in at least two of three tissue cores. Human testicular germ cell tumor tissue was used as a positive control for LIN28 staining, while sections processed in parallel without primary antibodies were used as negative controls. All immunohistochemical stains were scored independently by TS, DP, MB and NH, and reviewed by $\mathrm{AH}$ and $\mathrm{CH}$.

Gene expression and methylation array data analyses

Gene expression data from 59 primary CNS-PNETs arising in different locations were generated using the Illumina HT-12v4 arrays and normalized as previously described [17]. Methylation data generated using the Illumina human $450 \mathrm{k}$ arrays for 45 primary CNS-PNETs were background-normalized in Genome Studio (v. 2011.1) to obtain beta values for downstream analyses. All X and Y chromosome probes ( $n=11,649)$, single-nucleotide polymorphisms (dbSNP, $n=88,679$ ), and unannotated probes (relative to hg19, $n=65$ ) were excluded leaving a total of 385,184 probes for methylation analyses. Genes and probes were ranked by largest coefficient of variation and standard deviation, respectively. Unsupervised hierarchical clustering (HCL; Partek Genomics Suite, v6.6) of gene expression and methylation data were, respectively, established using Pearson's Dissimilarity performed iteratively on 200-2,000 genes and 200-10,000 probes to identify a minimal gene set associated with the most stable gene expression and methylation tumor cluster patterns (Supplemental Fig. 2).

\section{Statistical analyses}

Median age at diagnosis was compared using KruskalWallis test and Log Rank (Mantel-Cox) analysis was used to assess survival. All other clinical and biological characteristics were compared using Pearson Chi-Square $\left(\chi^{2}\right)$. A $p$ value of $<0.05$ was regarded as significant for all analyses. All statistical analyses were done using SPSS version 22.0.

\section{Cell culture and growth assays}

To generate stable tumor cell line, A664, a pre-treatment primary tumor from a non-C19MC amplified/LIN28+ CNS-PNET (PNET398) was dissociated by gentle manual trituration followed by passage through a $40-\mu \mathrm{m}$ mesh filter. Cells were grown in low-adhesion tissue culture flasks (Sarstedt, Montreal, QC, CA) in defined serum-free media at $37{ }^{\circ} \mathrm{C}, 5 \% \mathrm{CO}_{2}$. Culture media consisted of human neural stem cell proliferation media (Stem Cell Technologies, Vancouver, BC, CA) supplemented with heparin, epidermal growth factor (EGF, $10 \mathrm{ng} / \mathrm{ml}$, Sigma Aldrich, St. Louis, MO, USA), and fibroblast growth factor (FGF, $10 \mathrm{ng} /$ ml, Stem Cell Technologies, Vancouver, BC, CA). Cells were dissociated using Accumax (Millipore, MA, USA) by manual trituration and re-plated at $\sim 40 \%$ confluency $(20,000$ cells $/ \mathrm{ml})$ and maintained stably for $>10$ consecutive passages.

For drug treatment and growth assays, A664 cells were plated in 96-well dishes at 2,000 cells/well and incubated overnight. Cells were then treated with rapamycin (Sigma Aldrich, St. Louis, MO, USA), 5-azacytidine (Sigma Aldrich, St. Louis, MO, USA) or vorinostat (Selleckchem, Burlington, ON, CA) and evaluated for changes in cell proliferation assays at various days post-treatment using CellTiter $96^{\circledR}$ AQueous One Solution Cell Proliferation Assay kit (Promega, Madison WI, USA). Viable cell numbers were determined based on absorbance at $575 \mathrm{~nm}$ using a Versamax microplate reader (Molecular Devices, Sunnyvale, CA, USA). 


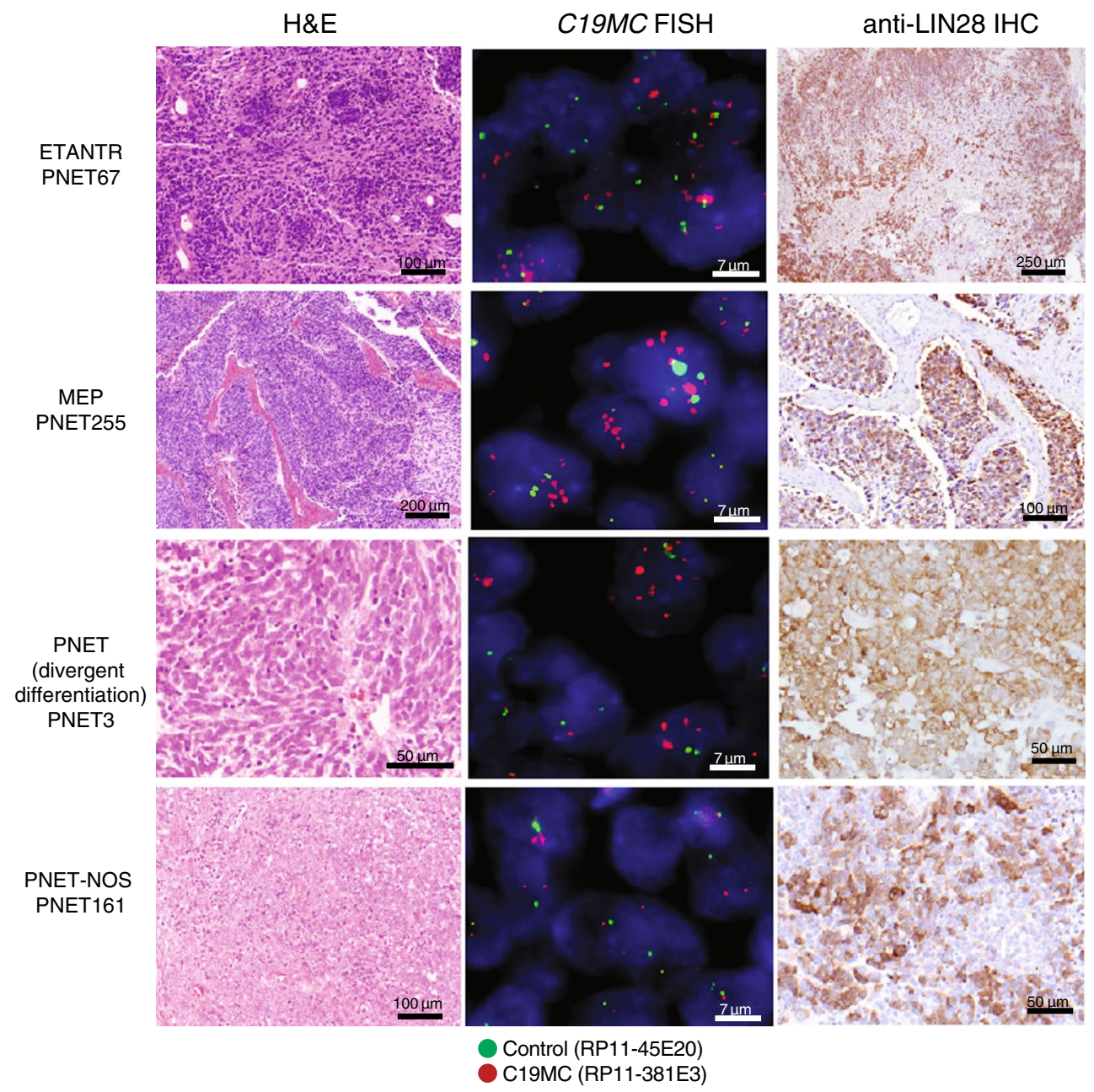

Fig. 1 Histologic spectra of CNS-PNETs with C19MC amplification and LIN28 immunopositivity. Representative $\mathrm{H}$ and $\mathrm{E}$ stains, C19MC FISH and LIN28 IHC analyses of CNS-PNETs with histologic fea-

Quantitative real-time (qRT-PCR), western blot and siRNA knockdown analyses

For LIN28 knockdown, A664 cells were plated at a density of $1.5 \times 10^{5}$ cells $/ \mathrm{ml}$ in 6 -well plates. Scrambled control or LIN28-specific siRNA (Fisher Scientific, Ottawa, ON, CA) was transiently transfected into cells using Lipofectamine 2000 (Life Technologies, Burlington, ON, CA) as per the manufacturer's protocol. Cells were harvested at 48-h posttransfection for protein extraction and analysis by Western blotting. Western blot analysis and chemiluminescence detection were performed using standard protocols. Secondary HRP-linked donkey anti-Rabbit or HRP-linked sheep anti-Mouse (\#NAV934, 931) was from GE Healthcare (Baie-d'Urfe, QC, CA).

For miRNA-specific qRT-PCR, single-stranded cDNA was synthesized from $10 \mathrm{ng}$ of RNA using a miR-specific stem-loop reverse-transcription RT-primer and the tures of ETANTR (PNET67), MEP (PNET255), CNS-PNET with divergent differentiation (PNET3) and undifferentiated PNET-NOS (PNET161)

TaqMan ${ }^{\circledR}$ MicroRNA Reverse Transcription Kit (Life Technologies, Burlington, ON, CA). qRT-PCR was performed using TaqMan ${ }^{\circledR}$ Universal PCR Master Mix, no AmpErase ${ }^{\circledR}$ UNG (Life Technologies, Burlington, ON, CA) according to the manufacturer's instructions; miRNA expression levels were normalized relative to RNU6B using the $\Delta C_{\mathrm{t}}$ method, and compared to that of 16-week-old whole human fetal brain control.

\section{Results}

CNS-PNETs with C19MC amplification and LIN28 immunopositivity span a histologic and anatomic spectrum of tumors

We $[13,17]$ and others $[10,11]$ previously reported frequent amplification of the C19MC oncogenic miRNA 

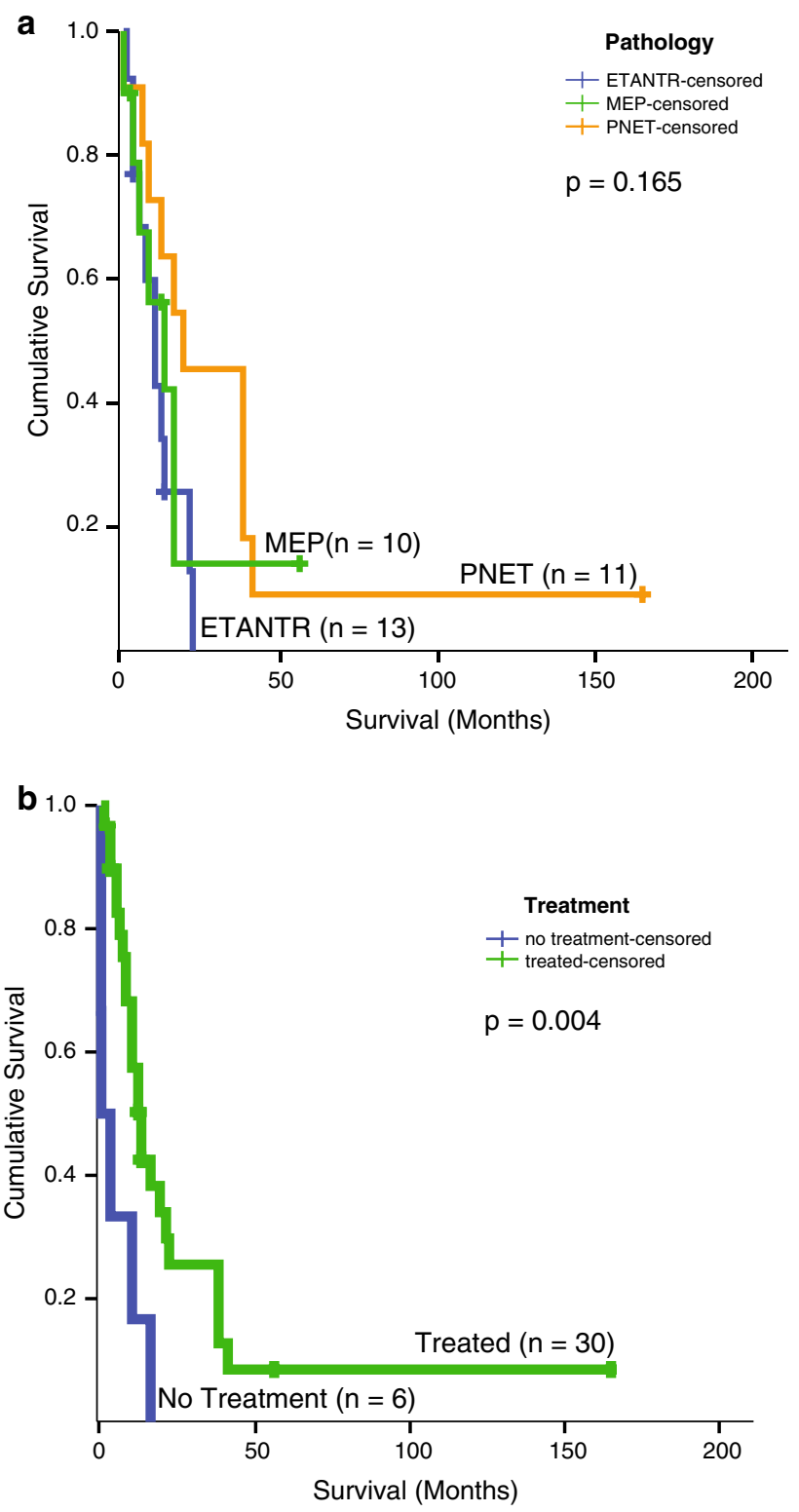

Fig. 2 Survival analyses of $C 19 M C$ amplified and/or LIN28+ CNSPNETs. a Log-rank survival analysis stratified by histologic classes of CNS-PNETs; log-rank test comparing overall survival of ETANTR vs MEP vs PNET is shown. b Log-rank comparison of overall survival for all CNS-PNETs stratified by receipt of treatment versus no treatment

cluster and high LIN28 protein expression in an aggressive sub-group of CNS-PNETs. To comprehensively define the clinico-pathologic features of these newly recognized molecular entities, we first tested the diagnostic robustness of the C19MC and LIN28 markers in a spectrum of pediatric brain tumors for which materials were available for central re-review of histopathology and confirmation of histologic diagnosis according to the current WHO CNS tumor classification criteria. These comprised an institutional cohort of $128 \mathrm{MBs}$, 45 ATRTs, 105 EPNs, 50 HGGs,
20 CPCs, and 103 CNS-PNETs from various institutions; 450 and 416 samples had materials available for FISH and LIN28 IHC analyses. As reported previously, C19MC amplification or copy number gains were restricted to and detected in $24.3 \%(25 / 103)$ of CNS-PNETs, while LIN28 immunopositivity was observed in $21.4 \%(22 / 103)$ of all CNS-PNETs. However, in contrast to C19MC amplification, cytoplasmic LIN28 immunopositivity was not restricted to CNS-PNETs but was also observed in a spectrum of other tumors including $24.4 \%$ (11/45) of ATRTs and $19.5 \%$ (8/41) of HGGs (Supplemental Table 1). These results indicate that LIN28 immunopositivity alone may not be sufficient to establish a diagnosis of CNS-PNETs.

To evaluate the relationship of CNS-PNETs with C19MC amplification and LIN28 positivity to known histologic sub-types of CNS-PNETs [14], we next conducted more detailed evaluation of tumor histopathology and clinical features in an expanded cohort of 54 CNS-PNETs identified on the basis of C19MC amplification and/or LIN28 expression (Table 1, Supplemental Table 2). Interestingly, of 45 tumors with complete histopathologic review, 22/45 (49\%) exhibited ependymoblastic rosettes with neuropil consistent with a histologic diagnosis of ETANTR (including 3 tumors initially diagnosed as EPBs), 12/45 (26.7 \%) displayed papillary features characteristic of MEP with variable amounts of neuropil and rosette formation, while a subset of 11/45 (24\%) tumors lacked characteristic histologic features of ETANTRs, EPBs or MEPs but exhibited variable differentiation or relatively bland histology compatible with classic or CNS-PNET-NOS (Fig. 1). In addition to spanning histologic sub-classes, we observed that C19MC amplified/LIN28+ CNS-PNETs also exhibited multiple CNS locations; cerebral origins were most common $(41 / 54 ; 76 \%)$, but $24 \%(13 / 54)$ arose in other CNS locations including the cerebellum $(6 / 54 ; 11 \%)$, brain stem $(5 / 54 ; 9 \%)$, pre-sacral space $(1 / 54 ; 2 \%)$ and optic nerve $(1 / 54 ; 2 \%)$. Of note, $5 / 37$ (14\%) tumors with overlapping data on C19MC and LIN28 status exhibited LIN28 positivity only, without changes in $C 19 M C$ expression or copy number; these tumors were found in each histologic class (please see Supplementary Table 2 for details).

Analyses of clinical features showed that 24/37 (65\%) of tumors were non-metastatic at diagnosis and arose predominantly in young children with a median age of 29 months at diagnosis. The incidence of tumor metastases, patient demographics (gender and age) and overall survival did not differ significantly across histologic classes. Detailed survival data available for 36/54 patients indicated a similar highly aggressive course with rapid disease progression and death for a majority of patients regardless of tumor histologic class (Fig. 2a). The median survival for all patients was only $12 \pm 1.9$ months (range of $0-165$ months). However, patients who received treatment had significantly longer 

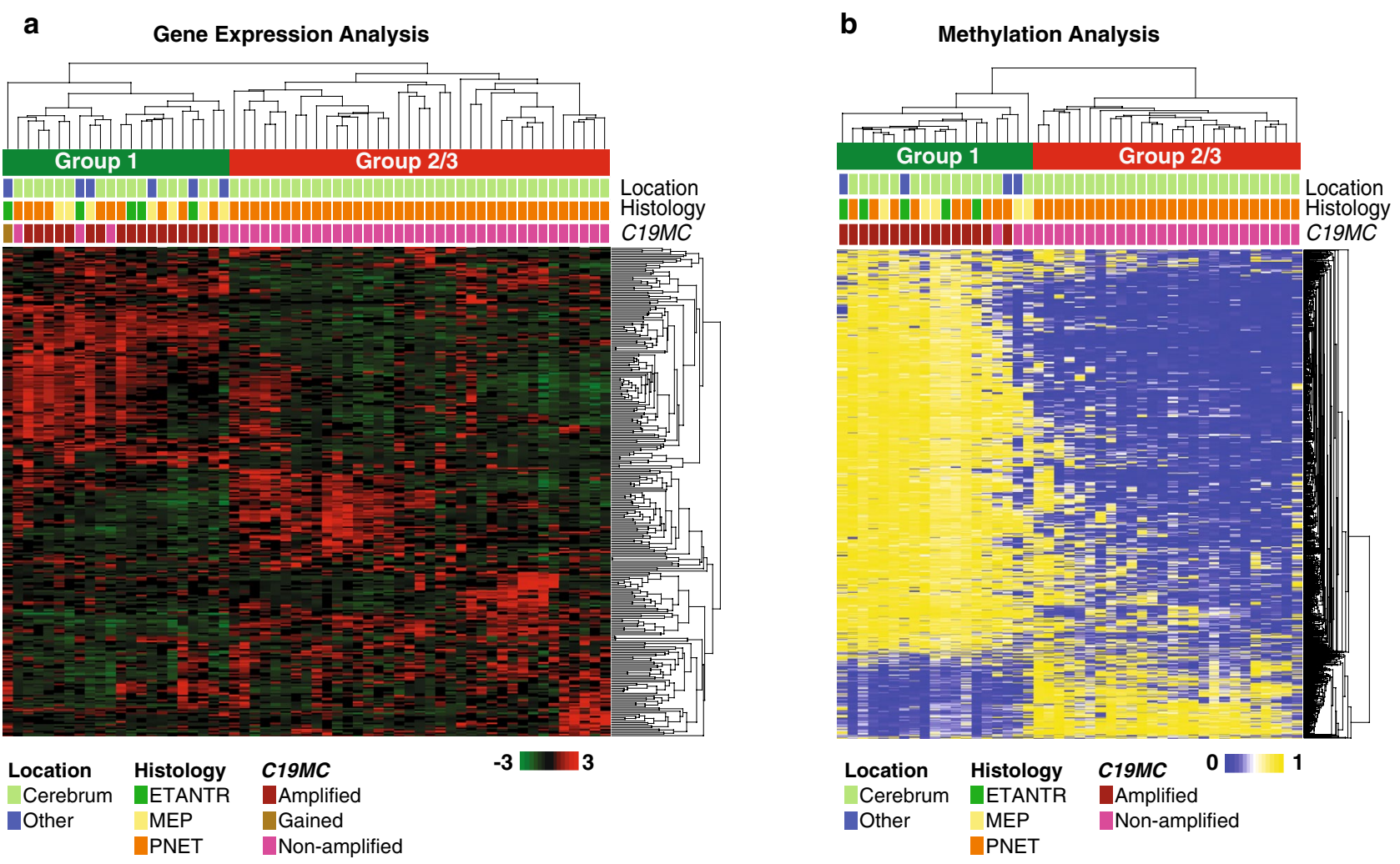

C qRT-PCR

miRNAs $\square$ 512-3p $\square$ 517a $\square$ 517c $\square$ 519a $\square$ 520g

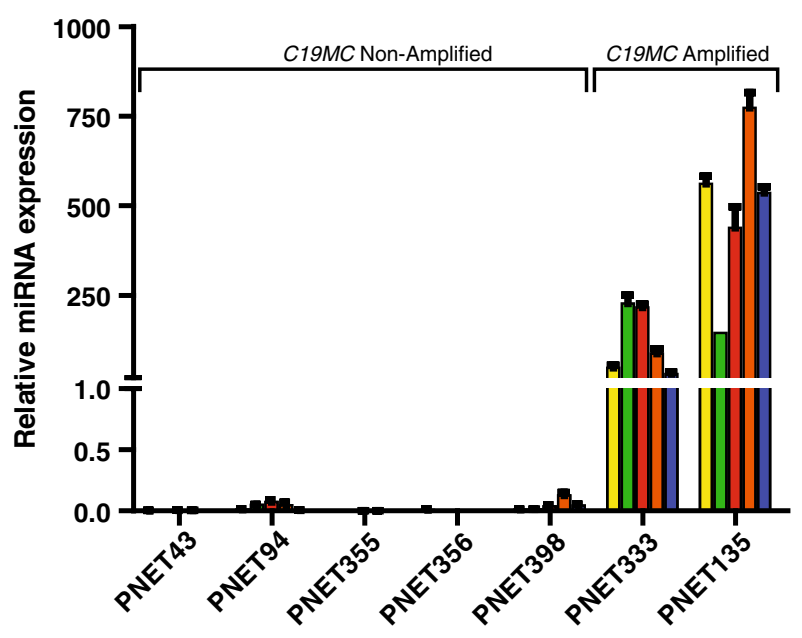

survival as compared to untreated patients (median survival of $13 \pm 1.9$ versus $0.060 \pm 1.84$ months; Fig. 2b). Notably, six patients ( 5 with specific treatment information) had substantially longer survival of 38-204 months; three patients remained alive at 56, 165 and 204 months (Supplemental Table 2). These findings suggest that chemotherapy \pm radiotherapy treatment may benefit a subset of patients. d

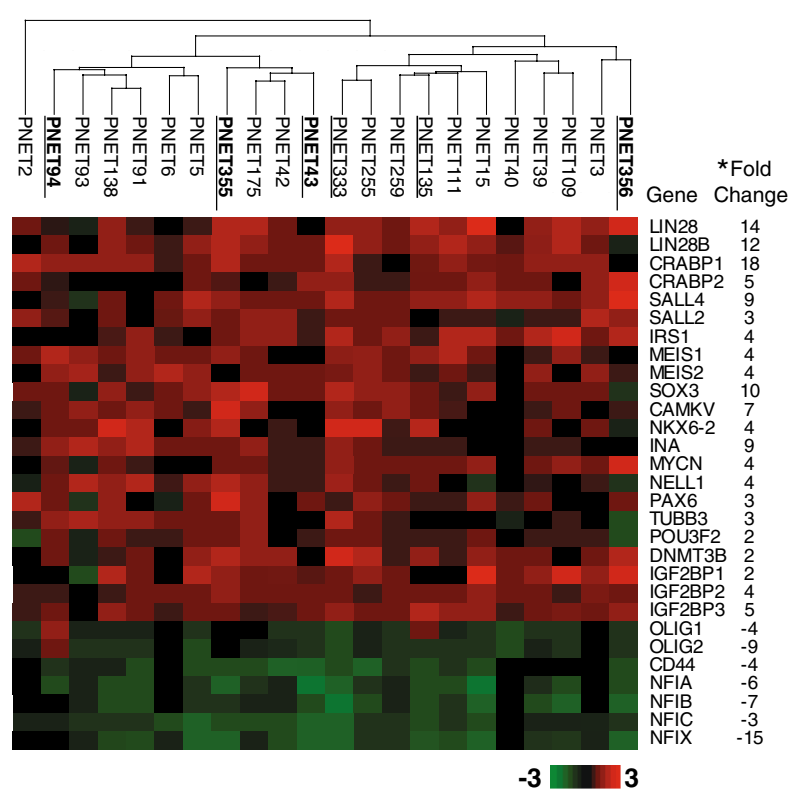

C19MC amplified/LIN28+ CNS-PNETs with various locations and histology exhibit common genetic and epigenetic signatures

We had previously demonstrated using global gene expression profiles that C19MC amplified/LIN28+ CNS-PNETs arising in the cerebral hemispheres comprised a molecular 
\Fig. 3 Unified gene expression and methylation signatures for C19MC amplified and/or LIN28 + CNS-PNETs. a, b Unsupervised hierarchical cluster analysis was performed on gene expression (a) and methylation data (b) generated, respectively, from 59 (Illumina HT-12v4 arrays) and 45 (Illumina human $450 \mathrm{k}$ arrays) primary CNS-PNETs. Most stable tumor cluster patterns were achieved with a minimal set of 300 genes, and across 500-4000 methylation probes (Supplemental Fig. 2) and indicated distinct segregation of C19MC amplified/LIN8+ tumors from Groups 2/3 CNS-PNETs, which lack either feature. Anatomic location of individual tumors, specific CNS-PNET histology and C19MC genomic status are indicated. c Quantitative RT-PCR analyses of C19MC miRNAs: miR-512-3p, $517 \mathrm{a}, 517 \mathrm{c}, 519 \mathrm{a}, 520 \mathrm{~g}$, in a subset of CNS-PNETs without $C 19 M C$ genomic amplification is shown relative to that of $C 19 M C$ amplified tumors, RNU6B served as control. d Genes most highly enriched in group 1 versus group 2/3 CNS-PNETs were identified using a supervised $t$ test adjusted for multiple hypothesis testing (false discovery rate $\leq 0.05$ ). Heat map shows relative magnitude of enrichment for specific genes with functions in cell lineage, signaling and epigenomic regulation at a significance of $* q \leq 0.05$. Tumor analyzed by qRT-PCR in $\mathbf{c}$ are underlined, non-C19MC amplified tumors and the C19MC amplified control tumor analyzed are, respectively, shown in bold

class with relative enrichment of primitive neural features that was distinct from two other molecular groups (designated group 2/3) of hemispheric CNS-PNETs [17]. The convergence of clinical phenotypes observed in C19MC amplified/LIN28+ tumors with different histologic features and anatomic locations, prompted us to investigate whether all C19MC amplified/LIN28+ tumors, regardless of histology or location, comprised a common molecular entity. Indeed, unsupervised hierarchical cluster analyses of gene expression data from 22 C19MC amplified/LIN28+ tumors, which included five ETANTRs, seven MEPs, and ten PNETs from different CNS locations, clustered together as a common molecular class distinct from Group 2/3 CNSPNETs (Fig. 3a). Similarly, unsupervised cluster analyses of global methylation profiles generated from an overlapping cohort of 19 C19MC amplified/LIN28+ tumors, comprised of 5 ETANTRs, 5 MEPs, and 9 PNETs from various locations, showed they formed a common molecular cluster distinct from the group 2/3 hemispheric CNS-PNETs (Fig. 3b). In keeping with our prior studies of hemispheric CNS-PNETs [13, 17], C19MC amplified/LIN28+ tumors with different location and histology also exhibited a gene expression signature enriched for pluripotent and neural cell lineage genes. Notably, all tumors with or without C19MC amplification expressed high levels of pluripotent genes LIN28/LIN28B, and low levels of neural differentiation genes including the NF1 family of transcription factors (Fig. 3c, d). Collectively, our analyses indicate that all CNS-PNETs with C19MC amplification and/or LIN28 immunopositivity, regardless of histologic sub-class or location, share similar molecular and genetic makeup, thus indicating they comprise a common biological and diagnostic entity.
Candidate therapeutics for $C 19 M C$ amplified and/or LIN28+ CNS-PNETs

In recent studies, we showed that the LIN28/let7/P13KmTOR pathway is up-regulated and can be targeted by Rapamycin in a C19MC amplified primary ETANTR cell line [19]. In addition, we demonstrated that the unique methylation landscape of C19MC amplified tumors is in part imposed via $C 19 M C$-mediated repression of $R B L 2$, and consequent up-regulation of DNMT3B [9]. The unified molecular signature of LIN28+/C19MC amplified and non-amplified CNS-PNET indicated that they may share common therapeutic pathways. We observed that LIN28/LIN28B and P13K-mTOR components, IGFBP13 , which have been linked to a common functional pathway [20, 22], as well as DNMT3B were enriched in gene expression signatures of CNS-PNETs with or without C19MC amplification (Fig. 3d). Consistent with these observations, IHC analyses revealed up-regulation of phospho-S6 (pS6), an mTOR pathway target, as well as DNMT3B in C19MC amplified and non-amplified primary tumors (Fig. 4a, b).

To investigate whether pharmacologic inhibitors of mTOR and DNA methylation may have therapeutic roles in all group 1 CNS-PNETs, we tested the effects of rapamycin as well pharmacologic inhibitors of DNA methyl transferase function (5-azacytidine and vorinostat), on growth of a tumor cell line (A664) derived from a non-C19MC amplified primary cerebral PNET398. Using quantitative RT-PCR and siRNA-mediated knockdown of LIN28, we confirmed that the LIN28/let7/P13K-mTOR axis was conserved in A664 cells. Indeed, rapamycin treatment significantly inhibited A664 cell growth with concomitant down-regulation of pS6 expression (Fig. 5b-d). Similarly, we observed that 5-azacytidine and vorinostat significantly inhibited A664 cell growth in a dose-dependent manner (Fig. 5e, f). Collectively, these data highlight regulators of $\mathrm{P} 13 \mathrm{~K} / \mathrm{mTOR}$ signaling, as well epigenomic modifiers, as novel promising therapeutic targets for these recalcitrant infantile tumors.

\section{Discussion}

In prior studies, we identified three transcriptional classes of CNS-PNETs arising in the cerebral hemispheres. Specifically, we reported that amplification of the oncogenic C19MC miRNA locus and/or high expression of LIN28, a pluripotency gene, identified a distinctively aggressive sub-group of hemispheric tumors, which we called group 1 tumors [17]. In this study, we demonstrate that C19MC amplification and/or LIN28 expression are seen in CNSPNETs with a spectrum of histology and location, and 

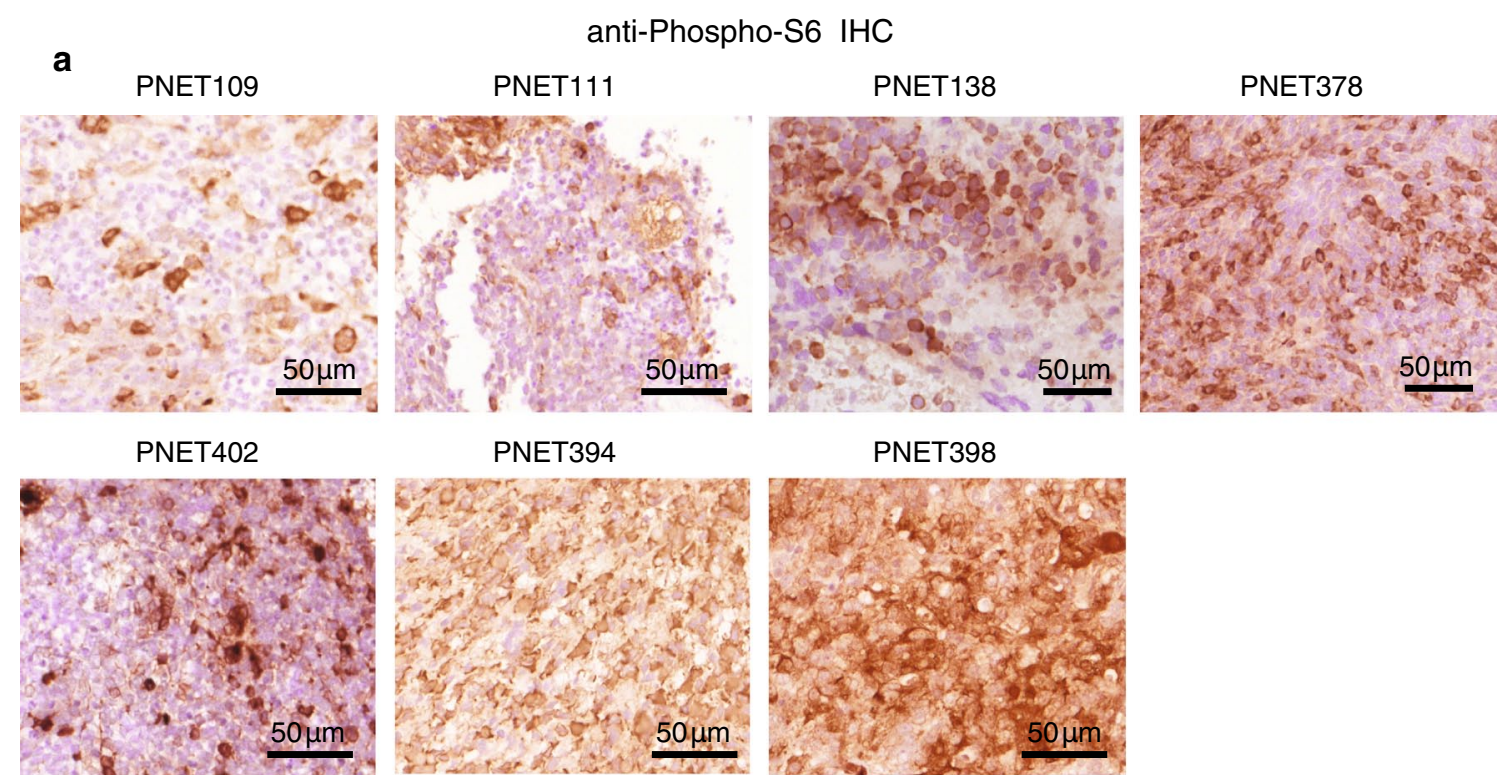

b

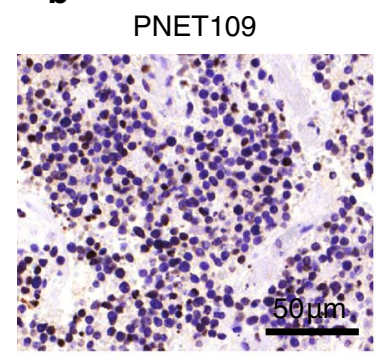

PNET402

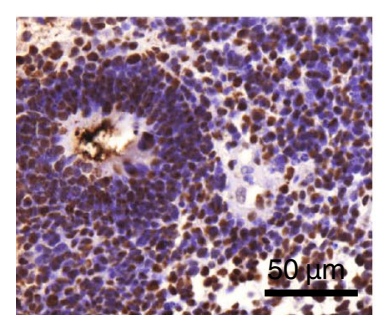

anti-DNMT3B IHC

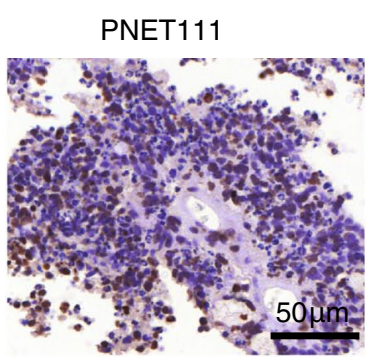

PNET394

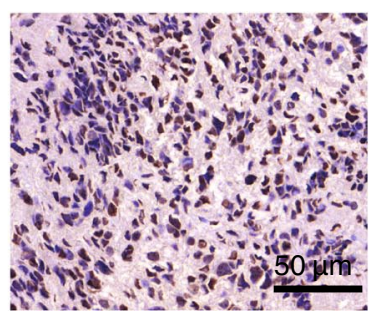

PNET138

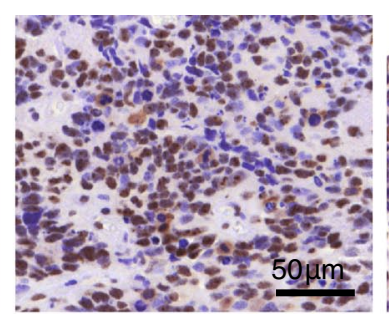

PNET398

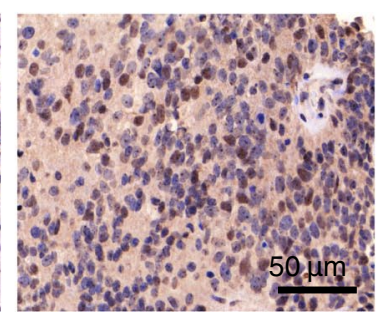

Fig. 4 Expression of mTOR target, phospho-S6 and DNA methyl transferase, DNMT3B, in group 1 CNS-PNETs. Representative a phospho-S6 and b nuclear DNMT3B immunostain patterns in
LIN28+ C19MC amplified (PNET 109, 111, 138, 378, 402) and nonamplified primary group 1 CNS-PNETs (PNET394 and 398)

number changes of $C 19 M C$ in any other malignant pediatric brain tumors with confirmed histopathologic diagnostic features of MBs, ATRTs, EPNs, HGGs, and CPCs. The pathogenic and diagnostic importance of this locus in CNS-PNETs is further highlighted by recent identification of TTYH1:C19MC gene fusions which is associated with very high expression of specific C19MC miRNAs and suggest $C 19 M C$ drives oncogenesis in part by facilitating maintenance and transformation of a very early, neural compartment [9]. Notably, although we observed cytoplasmic LIN28 expression uniformly (100\%) in C19MC 
a

PNET398

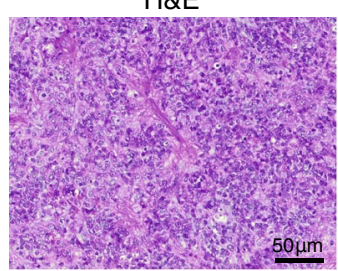

b

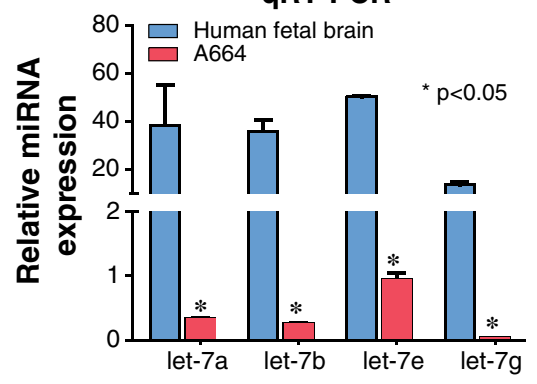

d

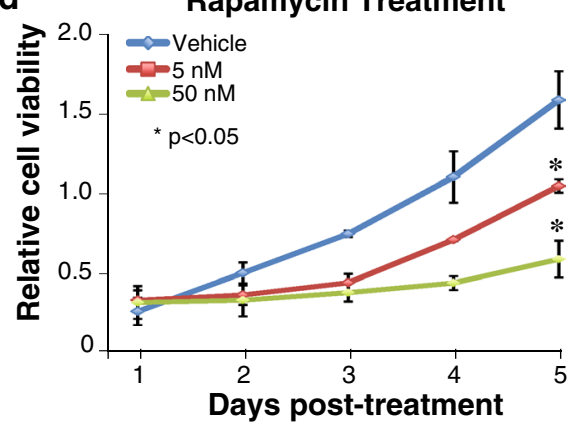

e

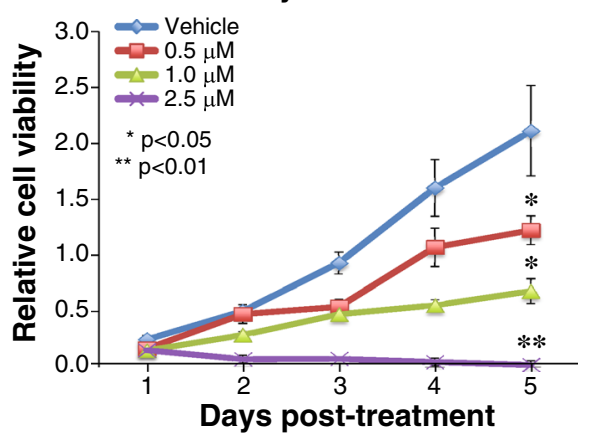

Fig. 5 Treatment of group 1 CNS-PNET cells with rapamycin, 5-azacytidine and vorinostat. A stable cell line from a primary nonC19MC amplified/LIN28+ CNS-PNET was established and tested for sensitivity to inhibitors of mTOR signaling and epigenomic modifiers as described in methods. a H and E stains, C19MC FISH and LIN28 IHC analysis of primary PNET398 from which the A664 cell line was derived, indicating lack of $C 19 M C$ amplification and strong LIN28 immunopositivity. b-c Quantitative RT-PCR and Western blot analyses indicating an intact LIN28-let7/mTOR axis in A664 cells. Expression of let-7a, 7b, 7e and 7g miRNAs was determined relative to that in normal human 16-week-old fetal brain and normalized to
C19MC FISH

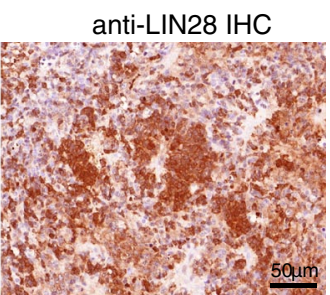

$7 \mu \mathrm{m}$

Control (RP11-45E20)

C19MC (RP11-381E3)

C

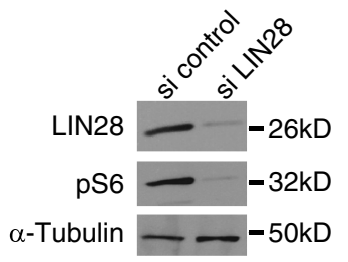

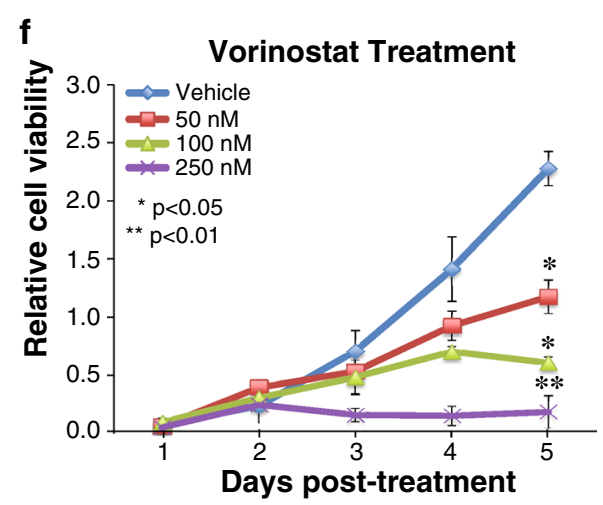

RNU6B. Results are shown as mean \pm SEM; $n=2$. A664 cells were treated with scrambled, control siRNA and siRNA directed against LIN28 and examined for expression of LIN28 and pS6 with $\alpha$-tubulin as loading control. d-f A664 cells were treated with varying doses of rapamycin, 5-azacytidine and vorinostat, and drug effect on cell viability was measured using MTS assays. Plots represent summary of three independent experiments with three replicas/data point; error bars represent SEM. * $p \leq 0.05$. Right panel in figure d shows Western blot analyses for LIN28 and pS6 expression in rapamycin-treated A664 cells; tubulin served as loading control 
amplified CNS-PNETs, our analyses also revealed cytoplasmic as well as nuclear LIN28 staining in a subset of MBs, ATRTs, EPNs and HGGs but not CPCs. Similar to prior reports of cytoplasmic LIN28 staining in 20-60\% of pediatric and adult gliomas [15] and $64 \%$ of ATRTs [3], we observed strong LIN28 cytoplasmic staining in up to 20-25\% of ATRTs and HGGs analyzed (Supplemental Tables 1,3), which contrasts with a report of cytoplasmic LIN28 immunostaining exclusively in ETANTRs [11]. The reason underlying these discrepant observations is unclear and may be related to the limitations of tissue microarray analyses to comprehensively capture tumor heterogeneity. Thus we propose that a combination of tumor morphology, together with cytoplasmic LIN28 immunostaining and C19MC genetic status, is needed to robustly distinguish group 1 CNS-PNETs or related embryonal tumors from other malignant pediatric brain tumors which may exhibit varying LIN28 expression.

Notably, as in our prior study [17], we observed that C19MC amplification or copy number gains together with high LIN28 expression identified CNS-PNETs that exhibited predominantly primitive neural histology with varying proportions of ependymoblastic rosettes, neural epithelium in papillary and pseudo-tubular formation, although such features were not necessarily identifiable in all C19MC amplified/LIN28+ CNS-PNET samples examined. We also observed that the proportion of cells with C19MC amplification and/or LIN28 expression varied across tumor samples that share group 1 CNS-PNETs molecular signatures, thus indicating that histopathologic analyses may be confounded by intra-tumoral heterogeneity which may reflect a varying, continuum of differentiation in the tumors. Indeed this may also apply to the histopathologic spectrum of ETANTRs, EPBs and MEPs seen in CNS-PNETs [7]. Although each of these entities are reported to be extremely rare, our data suggest that a combination of C19MC amplification and/or LIN28 expression together with careful morphologic assessment of tumor may identify up to $25 \%$ of CNS-PNETs that make up this histogenetic tumor spectrum. As these tumors predominantly arise in children $<4$ years of age, they may represent an even higher proportion of brain and other CNS tumors diagnosed in infancy. Thus, a diagnostic approach which combines histopathologic assessment together with evaluation of C19MC and LIN28 status will be important for capturing the true spectra of this disease. Therefore, we suggest that evaluation of C19MC and LIN28 status should be considered for all malignant neuroepithelial tumors arising in young children, regardless of CNS locations, in a manner similar to the diagnostic work-up for ATRTs.

We did not observe significant differences in C19MC amplification and LIN28 expression status nor in global identifiers based on gene expression and methylation analyses between various histological sub-types of CNS-PNETs. In addition, though trends toward older age and lower incidence of metastasis at diagnosis were observed in tumors with a PNET or variant PNET diagnosis, no significant differences were evident between histological subgroups. These subtle distinctions may prove to be clinically relevant upon analysis of a larger tumor cohort. Our data suggest that the majority of these tumors are localized at presentation, however, interestingly a subset of patients exhibited unusual patterns of metastasis including dural invasion and spread to extra-neural sites (Supplemental Table 2). Comprehensive diagnostic evaluation of large unbiased cohorts will be important for revealing disease patterns to inform and unify diagnostic work-up and therapeutic approaches for these rare tumors.

Consistent with prior studies [11, 17], we observed dismal overall survival in our study cohort with only $3 / 36$ patients who remain alive 56-204 months after diagnosis. Our data suggest a survival benefit for a small proportion of patients treated with chemotherapeutic regimes, with or without radiotherapy and underscore the need for better therapies in this disease. Our recent [19] and current study which demonstrates that rapamycin, a PI3K/mTOR inhibitor, significantly inhibits growth of cell lines derived from both $C 19 M C$ amplified and non-amplified primary group 1 tumors suggests targeting the $\mathrm{P} 13 \mathrm{~K} / \mathrm{mTOR}$ pathway as a novel therapeutic avenue for this disease. In this study, we also demonstrated that 5-azacytidine, a pharmacological antagonist of DNMTs, and HDAC inhibitor, vorinostat, had significant effects on A664 cell growth. Together with recent demonstration that C19MC regulates the RBL2DNMT3B axis [9], our data suggest epigenetic regulators as important new therapeutic targets in this disease.

In summary, our study, together with a recent similar report by Korshunov et al. [12], indicate CNS-PNETs with $C 19 M C$ amplification and/or LIN28 expression span various histologies but comprise a single molecular disease that warrant common therapeutic strategies. Our study provide novel insights into potential targetable pathways for this frequently fatal but relatively uncommon disease and report on a unique cell line model that will be an invaluable resource for future therapeutic investigations.

Acknowledgments This project was funded by grants from the Canadian Institute of Health Research (Grant No. 102684) and b.r.a.i.n.child to AH. Technical contributions from Rene Head and Jim Loukides are gratefully acknowledged.

Open Access This article is distributed under the terms of the Creative Commons Attribution License which permits any use, distribution, and reproduction in any medium, provided the original author(s) and the source are credited. 


\section{References}

1. Adamek D, Sofowora KD, Cwiklinska M, Herman-Sucharska I, Kwiatkowski S (2013) Embryonal tumor with abundant neuropil and true rosettes: an autopsy case-based update and review of the literature. Childs Nerv Syst 29:849-854. doi:10.1007/ s00381-013-2037-4

2. Buccoliero AM, Castiglione F, Rossi Degl'Innocenti D et al (2010) Embryonal tumor with abundant neuropil and true rosettes: morphological, immunohistochemical, ultrastructural and molecular study of a case showing features of medulloepithelioma and areas of mesenchymal and epithelial differentiation. Neuropathology 30:84-91. doi:10.1111/j.1440-1789.2009.01040.x

3. Deisch J, Raisanen J, Rakheja D (2011) Immunohistochemical expression of embryonic stem cell markers in malignant rhabdoid tumors. Pediatr Dev Pathol 14:353-359. doi:10.2350/10-09-0902-OA.1

4. Eberhart CG, Brat DJ, Cohen KJ, Burger PC (2000) Pediatric neuroblastic brain tumors containing abundant neuropil and true rosettes. Pediatr Dev Pathol 3:346-352

5. Gessi M, Giangaspero F, Lauriola L et al (2009) Embryonal tumors with abundant neuropil and true rosettes: a distinctive CNS primitive neuroectodermal tumor. Am J Surg Pathol 33:211-217. doi:10.1097/PAS.0b013e318186235b

6. Hoos A, Cordon-Cardo C (2001) Tissue microarray profiling of cancer specimens and cell lines: opportunities and limitations. Lab Invest 81:1331-1338

7. Judkins AR, Ellison DW (2010) Ependymoblastoma: dear, damned, distracting diagnosis, farewell!*. Brain Pathol 20:133139. doi:10.1111/j.1750-3639.2008.00253.x

8. Kallioniemi OP, Wagner U, Kononen J, Sauter G (2001) Tissue microarray technology for high-throughput molecular profiling of cancer. Hum Mol Genet 10:657-662

9. Kleinman CL, Gerges N, Papillon-Cavanagh S et al (2014) Fusion of TTYH1 with the C19MC microRNA cluster drives expression of a brain-specific DNMT3B isoform in the embryonal brain tumor ETMR. Nat Genet 46:39-44. doi:10.1038/ ng. 2849

10. Korshunov A, Remke M, Gessi M et al (2010) Focal genomic amplification at $19 \mathrm{q} 13.42$ comprises a powerful diagnostic marker for embryonal tumors with ependymoblastic rosettes. Acta Neuropathol 120:253-260. doi:10.1007/s00401-010-0688-8

11. Korshunov A, Ryzhova M, Jones DT et al (2012) LIN28A immunoreactivity is a potent diagnostic marker of embryonal tumor with multilayered rosettes (ETMR). Acta Neuropathol 124:875881. doi:10.1007/s00401-012-1068-3
12. Korshunov A, Sturm D, Ryzhova M et al (2013) Embryonal tumor with abundant neuropil and true rosettes (ETANTR), ependymoblastoma, and medulloepithelioma share molecular similarity and comprise a single clinicopathological entity. Acta Neuropathol. doi:10.1007/s00401-013-1228-0

13. Li M, Lee KF, Lu Y et al (2009) Frequent amplification of a chr19q13.41 microRNA polycistron in aggressive primitive neuroectodermal brain tumors. Cancer Cell 16:533-546. doi:10.1016/j.ccr.2009.10.025

14. Louis DN, International Agency for Research on Cancer (2007) WHO classification of tumours of the central nervous system. International Agency for Research on Cancer, France

15. Mao XG, Hutt-Cabezas M, Orr BA et al (2013) LIN28A facilitates the transformation of human neural stem cells and promotes glioblastoma tumorigenesis through a pro-invasive genetic program. Oncotarget 4:1050-1064

16. Nobusawa S, Yokoo H, Hirato J et al (2012) Analysis of chromosome 19q13.42 amplification in embryonal brain tumors with ependymoblastic multilayered rosettes. Brain Pathol 22:689-697. doi:10.1111/j.1750-3639.2012.00574.x

17. Picard D, Miller S, Hawkins CE et al (2012) Markers of survival and metastatic potential in childhood CNS primitive neuro-ectodermal brain tumours: an integrative genomic analysis. Lancet Oncol 13:838-848. doi:10.1016/S1470-2045(12)70257-7

18. Ray A, Ho M, Ma J et al (2004) A clinicobiological model predicting survival in medulloblastoma. Clin Cancer Res 10:76137620. doi:10.1158/1078-0432.CCR-04-0499

19. Spence T, Perotti C, Sin-Chan P et al (2014) A novel C19MC amplified cell line links Lin28/let-7 to mTOR signaling in embryonal tumor with multilayered rosettes. Neuro-oncology 16:62-71. doi:10.1093/neuonc/not162

20. Suvasini R, Shruti B, Thota B et al (2011) Insulin growth factor-2 binding protein 3 (IGF2BP3) is a glioblastoma-specific marker that activates phosphatidylinositol 3-kinase/mitogen-activated protein kinase (PI3 K/MAPK) pathways by modulating IGF-2. J Biol Chem 286:25882-25890. doi:10.1074/jbc.M110.178012

21. Woehrer A, Slave I, Peyrl A et al (2011) Embryonal tumor with abundant neuropil and true rosettes (ETANTR) with loss of morphological but retained genetic key features during progression. Acta Neuropathol 122:787-790. doi:10.1007/s00401-011-0903-2

22. Zhu H, Shyh-Chang N, Segre AV et al (2011) The Lin28/ let-7 axis regulates glucose metabolism. Cell 147:81-94. doi:10.1016/j.cell.2011.08.033 